\title{
Single Walled Carbon Nanotube Sensor Platform for the Study of Extracellular Analytes
}

\author{
Joseph A. Stapleton, Eric M. Hofferber, Jakob Meier, Ivon Acosta Ramirez, and Nicole M. Iverson* \\ Department of Biological Systems Engineering, Institute of Agriculture and Natural Resources, College of Engineering, \\ University of Nebraska-Lincoln, 251 L. W. Chase Hall, P.O. Box 830726, Lincoln, NE 68583-0726 *iverson@unl.edu
}

\section{SUPPORTING INFORMATION}

Table s1: Atomic percent values are for each derivatization step necessary to produce avidin derivatized substrates. Carbon percentage increases with each step in the derivatization process indicating the presence of 3-GPTMS and avidin. Significant levels of nitrogen are not observed until avidin derivatization. Interestingly, silicon content increases upon 3-GPTMS functionalization presumably due to the Si atom present in 3-GPTMS. Oxygen content decreases following each step of derivatization in accordance with shielding effects.

\begin{tabular}{lcccc}
\hline & \multicolumn{4}{c}{ Atomic Percent } \\
Derivatization Step & C1s & N1s & Si2p & O1s \\
Piranha & 8.95 & 0.68 & 26.91 & 63.46 \\
Epoxy Silane & 16.75 & 0.67 & 57.07 & 25.5 \\
Avidin & 54.14 & 12.93 & 26.27 & 6.66 \\
\hline
\end{tabular}

Table s2: Atomic percent values are tabulated for every derivatization step. Carbon percentage is seen to increase with the addition of APTES. Nitrogen does not make up a significant percentage until APTES derivatization reinforcing its presence on the surface. Oxygen content decreases following ATPES derivatization in good accordance with shielding effects

\begin{tabular}{lllll}
\hline & \multicolumn{4}{l}{ Atomic Percent } \\
Derivatization Step & C1s & N1s & Si2p & 01s \\
Piranha & 8.95 & 0.68 & 26.91 & 63.46 \\
APTES & 15.77 & 2.98 & 25.74 & 55.51 \\
\hline
\end{tabular}




\begin{tabular}{|l|l|l|}
\hline & Biotinylated SWNT & Non-Biotinylated SWNT \\
\hline Sample 1 & 33.58 & 31.55 \\
\hline Sample 2 & 33.42 & 31.16 \\
\hline Sample 3 & 32.25 & 32.40 \\
\hline Average & 33.08 & 31.70 \\
\hline Standard Error & 0.42 & 0.37 \\
\hline
\end{tabular}

Table s3: Full width half max values, displayed graphically in Figure 2, are provided.

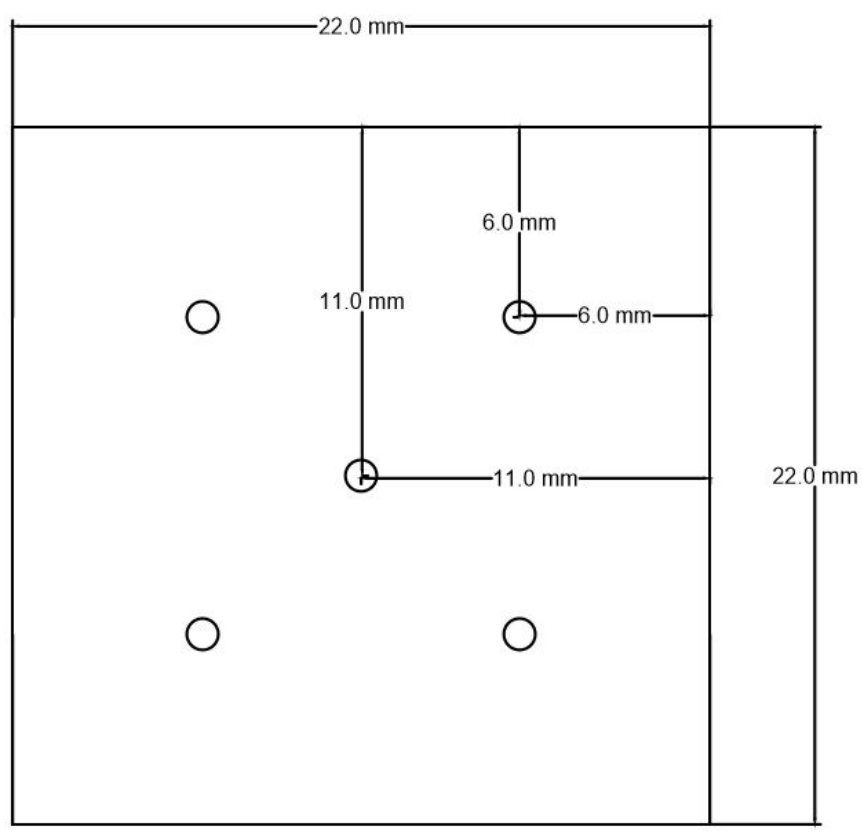

Figure s1: A $22 \times 22 \mathrm{~mm}$ section of glass slide was used. The figure demonstrates the locations imaged in the treated area where circles are the locations imaged. 5 subsamples per slide were always taken. Samples were moved in slightly from the edges to prevent edge effects from skewing results.

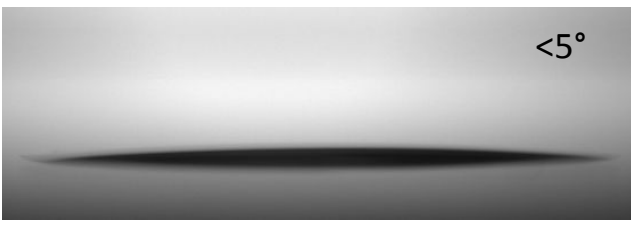

Figure s2: Glass slides were used as the base of the avidin and APTES treated slides. They had a contact angle of $<5^{\circ}$. The contact angle after each treatment step is shown in Figures 3 and $s 3$. 

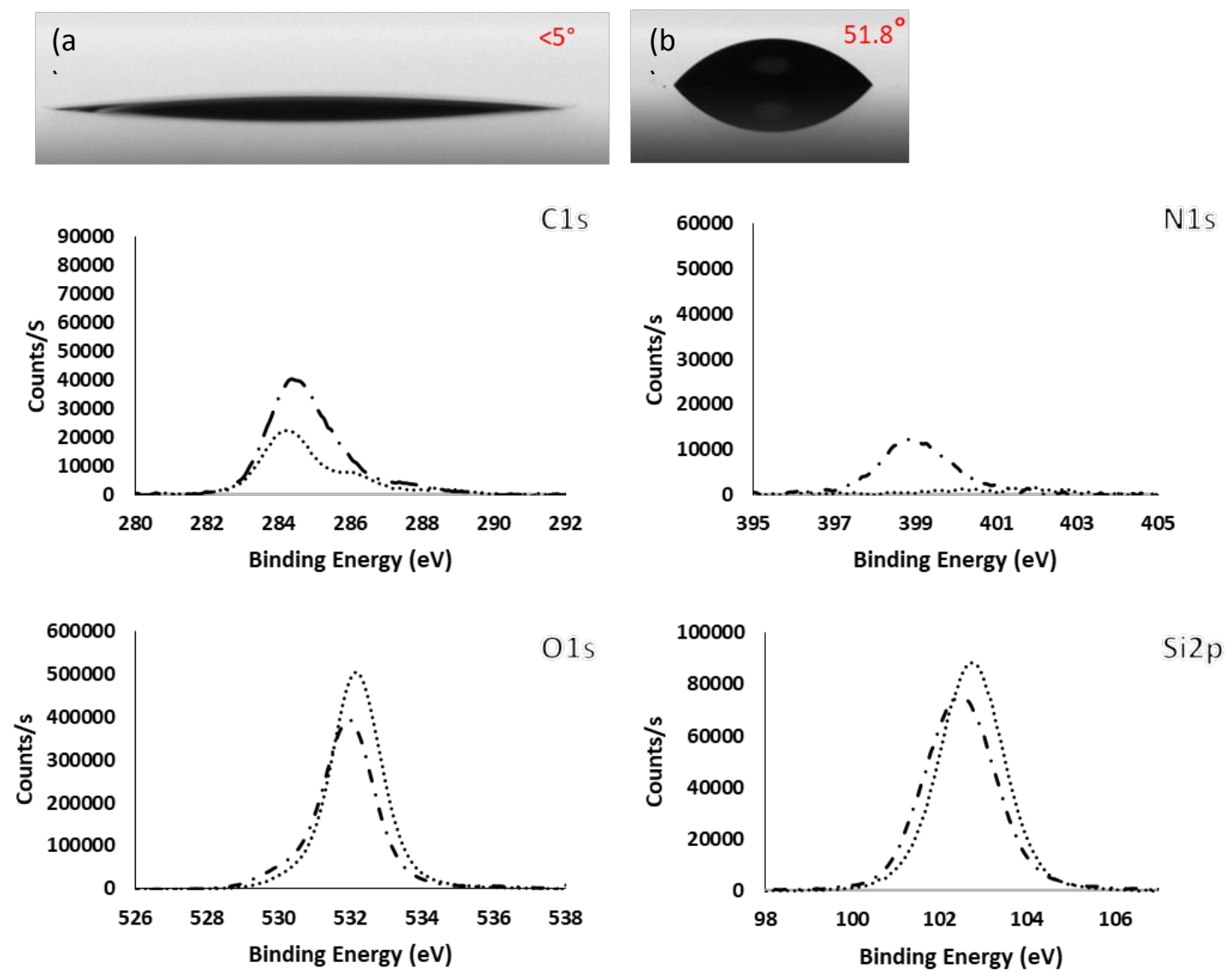

Figure s3: Generating APTES derivatized substrates (a) Piranha treated slides, demonstrated a contact angle of $<5^{\circ}$ which is consistent with glass substrates treated in this manner. (b) APTES treated slides, show a characteristic increase in contact angle of $51.8^{\circ}$. (XPS peaks) the C1s peak clearly demonstrates an increase in the total carbon content after APTES functionalization which can be attributed to the arm of the silane. The N1s peak showed no substantial peaks until APTES functionalization which can be attributed to the aminopropyl head of ATPES. Both the O1s and Si2p peaks demonstrate shielding effects as the addition of the silane reduces the signal intensity from the underlying $\mathrm{SiO}_{2}$ framework. 


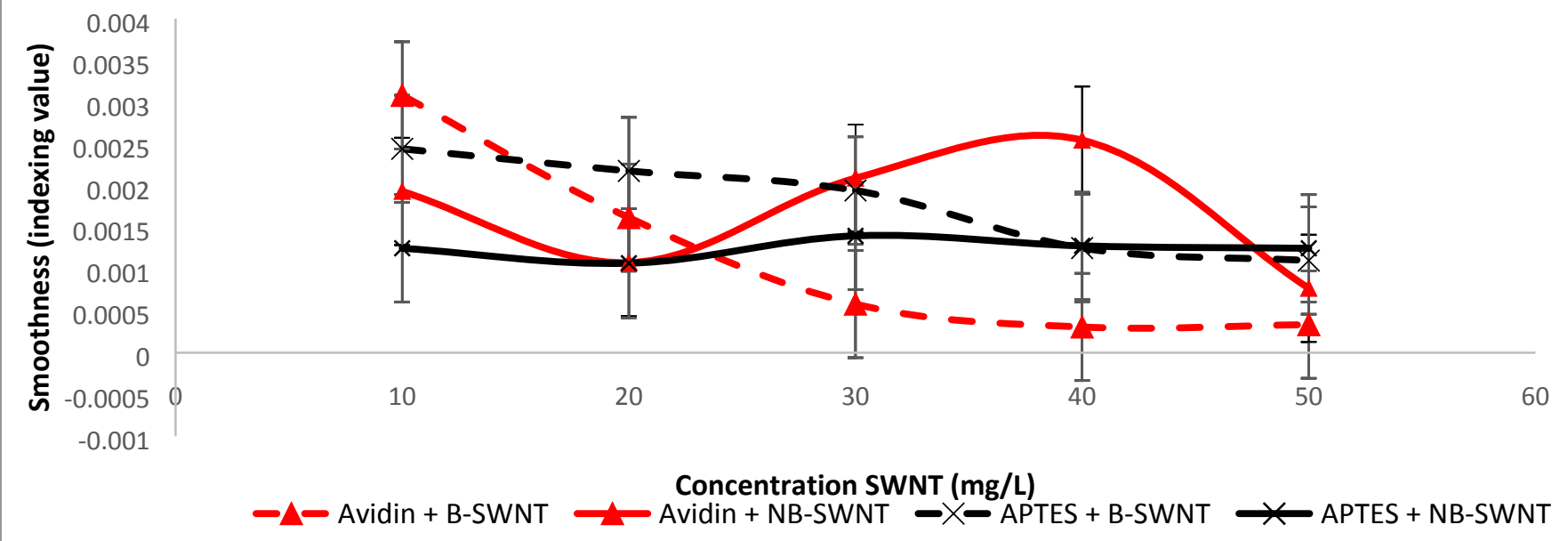

Figure s4: Another method of determining smoothness is to process the image with 1-level of 2D discrete wavelet decomposition; and then use the percent energy of the pixels being partitioned into the diagonal wavelet component as the index to assess randomness. Using this method, no significant differences in overall smoothness could be determine. 